\title{
EL SISTEMA DE COMUNICACIÓN INTEGRADO A LOS SISTEMAS DE GESTIÓN EN EL CONTEXTO ORGANIZACIONAL
}

\section{THE COMMUNICATION SYSTEM INTEGRATED TO THE MANAGEMENT SYSTEMS IN THE ORGANIZATIONAL CONTEXT}

\author{
Isis Leonor Suárez Caimary ${ }^{1}$ \\ Marianela Bermejo Salmon ${ }^{2}$ \\ Leonardo Charón Durive ${ }^{3}$
}

Recibido: 2021-09-20 / Revisado: 2021-10-25 / Aceptado: 2021-11-25 / Publicado: 2022-01-01

Forma sugerida de citar: Suárez-Caimary, I. L., Bermejo-Salmón, M. y Charón-Durive, L. (2022). El sistema de comunicación integrado a los sistemas de gestión en el contexto organizacional. Retos de la Ciencia. 6(12). 5161. https://doi.org/10.53877/rc.6.12.20220101.05

\section{RESUMEN}

La comunicación organizacional contribuye al cumplimiento de las funciones, tareas y/o actividades del puesto de trabajo en lo individual y en la general, a la coordinación del conjunto de actividades que se desarrollan en las entidades para la consecución de los resultados previstos. El presente trabajo tiene como objetivo la propuesta de un procedimiento para el diagnóstico y diseño del sistema de comunicación, integrada al sistema de dirección y gestión con enfoque estratégico. La metodología fue aplicada y validada en dos organizaciones de Santiago de Cuba con resultados satisfactorios. Se emplean diferentes técnicas y herramientas de investigación, que combinan el enfoque cuantitativo con el cualitativo, entre las que se encuentran:

\footnotetext{
${ }^{1}$ Ingeniera Química. Máster en Dirección. Investigador Agregado. Profesora Asistente. Centro de Información y Gestión Tecnológica, Santiago de Cuba-Cuba. E-mail: isis@megacen.ciges.inf.cu / https://orcid.org/0000-00026125-3912

${ }^{2}$ Licenciada en Economía. Máster en Administración de Negocios. Doctora en Ciencias Económicas. Profesora Titular. Facultad de Ciencias Económicas y Empresariales de la Universidad de Oriente. Santiago de Cuba-Cuba. E-mail: marianela@uo.edu.cu / https://orcid.org/0000-0001-6217-6480

${ }^{3}$ Ingeniero Mecánico. Master en Dirección. Especialista en Investigación, Desarrollo e Innovación. Centro de Información y Gestión Tecnológica. Santiago de Cuba-Cuba. E-mail: charon@megacen.ciges.inf.cu / https://orcid.org/0000-0002-3869-9297
} 
análisis bibliográfico, revisión y análisis documental, entrevistas en sus diversas tipologías, cuestionario en su forma de encuesta y listas de chequeo. Como resultado se logra desarrollar un procedimiento para el diagnóstico y diseño de la comunicación organizacional que imbrica los elementos teóricos, metodológicos, estructurales y prácticos con enfoque integrado a todos los sistemas de gestión proporcionando la mejora de los procesos empresariales. Su diseño a partir de las normativas técnicas y jurídicas vigente facilita su generalización y adaptabilidad a todo tipo de organización en función de sus características. La comunicación es un proceso que ocurre en cualquier tipo de organización involucrando permanentemente a los clientes internos y externos, tales como, trabajadores, directivos, clientes, proveedores y la sociedad.

Palabras clave: comunicación organizacional, gestión integrada, sistemas de gestión, diagnóstico de la comunicación

\section{ABSTRACT}

Organizational communication contributes to the fulfillment of the functions, tasks and /or activities of the job position individually and in general, to the coordination of the set of activities that are developed in the entities to achieve the expected results. Work objective is to propose a procedure for a diagnosis and design of the communication system, integrated into the direction and management system with a strategic approach. The methodology was applied and validated in two Santiago de Cuba's organizations with satisfactory results. Different research techniques and tools are used, combining the quantitative with the qualitative approach, among which are: bibliographic analysis, documentary review and analysis, differents interviews types, questionnaire in survey form and checklists. Is possible developing as a result procedure for a diagnosis and design of organizational communication that overlaps the theoretical, methodological, structural and practical elements with an integrated approach to all management systems, providing the improvement of business processes. Its design based on current technical and legal regulations facilitates its generalization and adaptability to all types of organizations depending on its characteristics. Communication is a process that occurs in any type of organization, permanently involving internal and external clients, such as workers, managers, clients, suppliers and society.

Keywords: organizational communication, integrated management, management systems, communication diagnosis

\section{INTRODUCCIÓN}

La aplicación de un sistema de comunicación en la empresa estatal socialista está estrechamente relacionada con la idea de solucionar los problemas. La comunicación contribuye al cumplimiento de las funciones, tareas y/o actividades del puesto de trabajo en lo individual y en la general, a la coordinación del conjunto de actividades que se desarrollan en las entidades para la consecución de sus objetivos con enfoque estratégico.

La comunicación organizacional se ha conocido desde los inicios de estos estudios con denominaciones diferentes como: comunicación organizacional, empresarial, institucional, organizativa, corporativa; todas referidas al mismo fenómeno (Núñez, 2010). 
En el Decreto 281/ 2007 Reglamento para la implantación y consolidación del Sistema de Dirección y Gestión Empresarial Estatal, articulo 671 se plantea:

La comunicación empresarial es el conjunto de actividades encaminadas a facilitar y agilizar el flujo (ida y vuelta) de mensajes que se dan entre los trabajadores entre sí y con su dirección, así como entre la empresa y su entorno. La aplicación de un correcto sistema de comunicación permite influir en las opiniones, aptitudes y conductas de los trabajadores de la empresa y los públicos externos, con el fin de cumplir con mayor eficiencia y rapidez los objetivos de la empresa.

La comunicación empresarial debe transmitir la situación existente en un determinado momento, de forma confiable, oportuna, eficaz, idónea, pertinente y con la calidad requerida. Debe realizarse en todos los sentidos y niveles de la estructura organizativa: ascendente, descendente, transversal y horizontal, y debe contribuir a la toma de decisiones.

Por tal motivo las organizaciones deben identificar y establecer los flujos, medios, canales, maneras y formas que les permitan trasmitir informaciones transparentes, confiables y oportunas de manera comprensible, que abarquen tanto a sus clientes internos como externos lo cual impactará en la mejora en cuanto a eficiencia y eficacia de sus procesos.

La comunicación es uno de los instrumentos estratégicos que contribuyen al mejoramiento continuo y al aumento de la productividad dada la influencia que tiene sobre la estimulación y el desempeño de los trabajadores, por cuanto, a través de su análisis y efectividad se logran identificar elementos clave que permiten elevar la calidad de vida laboral y la calidad de los productos o servicios que se ofertan a los clientes.

El primer paso para implantar el Sistema de Comunicación Organizacional acorde con el Decreto Ley 252/ 2007 es efectuar un diagnóstico. En la Guía de Autocontrol de la Contraloría General de la Republica para el componente Información y Comunicación, se refleja que el diagnóstico de comunicación:

a) Describe el enfoque de comunicación.

b) Define elementos de cultura organizacional, los valores compartidos, el sentido de pertenencia y aspectos de clima interno.

c) Detalla la naturaleza de los procesos de comunicación, redes, flujos, los canales más usados, el uso de los mensajes, frecuencia y su planificación.

d) Permite conocer conceptos de comunicación que se manejan en la entidad.

e) Precisa las características de directivos y trabajadores, sus expectativas, el grado de motivación, sus intereses y aspiraciones, así como la participación, entre otros aspectos.

f) Determina la relevancia y funciones de la comunicación en la empresa.

El sistema de comunicación empresarial, se encamina tanto a públicos internos, integrado por todos los trabajadores de las empresas, como a los públicos externos, conformados por el pueblo, los clientes, suministradores, competidores y por el resto de las instituciones gubernamentales o no gubernamentales que de una forma u otra se relacionan con la empresa; por lo que es indispensable el establecimiento de un sistema de comunicación para que no se pierda la coherencia entre las acciones que se realizan dentro de la empresa con la realidad del entorno (Dong, 2013).

Para que el sistema de comunicación diseñado resulte integrado al resto de los sistemas de dirección y gestión se requiere, además, identificar en el diagnostico el estado de los aspectos o requisitos pertinentes a cada uno y que tributan a la comunicación para la implementación eficaz de los mismos según las normas técnicas y jurídicas vigentes. Entre los sistemas se encuentran: 
- Gestión del Capital Humano: Ley 116/ 2013 y el Decreto 326/ 2014. Código de Trabajo y Reglamento del Código de Trabajo respectivamente.

- Control Interno: Resolución 60/ 2011. Normas del Sistema de Control Interno.

- Gestión de la Calidad: NC ISO 9001: 2015 Sistema de Gestión de la calidadRequisitos con orientación para su uso.

- Gestión Ambiental: NC ISO 14001: 2015 Sistema de Gestión AmbientalRequisitos con orientación para su uso.

- Gestión de la Seguridad y Salud en el Trabajo: NC ISO 45001: 2018 Sistema de Gestión de la Seguridad y salud en el trabajo-Requisitos con orientación para su uso.

- Gestión de la Innovación: NC ISO 56002: 2020 Gestión de la Innovación Orientación

Las NC ISO citadas anteriormente al estar alineadas, refieren en el requisito 7.4 lo concerniente a la comunicación para cada sistema de gestión, por lo que se requiere su inclusión en el sistema de comunicación organizacional, para que el mismo tenga enfoque integrado.

Bartoli en su libro Comunicación y Organización, sobre el sistema de comunicación afirma que, "organizar y comunicar desde una perspectiva de gestión supone a la vez determinar un marco estratégico firme y pilotar márgenes de maniobra interculturales o interpersonales" (1992, p. 35).

Llamas refiere que "un sistema de comunicación es aquel conjunto de elementos donde, mediante un emisor, mensaje y receptor, se produce un proceso comunicativo entre mínimo dos partes" (2021).

A partir de la revisión bibliográfica de los procedimientos para el diagnóstico del sistema de comunicación integrado, entre los que se encuentran Almirall. (2010) y Cirigliano. (2017), los autores del presente trabajo procedieron al diseño para la realización del mismo teniendo en cuenta que los existentes no contemplan la integración con los sistemas de gestión implementados atemperados a las normativas técnicas y jurídicas vigentes para la actividad de comunicación.

Por lo anterior se plantea como problema de investigación: ¿Qué procedimiento emplear para el diagnóstico y diseño del sistema de comunicación integrado a los sistemas de gestión en el contexto organizacional?

Y como objetivo general de este estudio: Diseñar un procedimiento para la realización del diagnóstico y diseño del sistema de comunicación integrado al resto de los sistemas de gestión organizacionales sobre la base de las normativas jurídicas y técnicas vigentes en el país.

Estructurado en fases, el procedimiento propuesto da respuesta a los requisitos de la legislación vigente en el país para la actividad de comunicación con enfoque estratégico, integrado y sistémico, lo cual permite su aplicabilidad, adaptabilidad y generalización en cualquier tipo de organización.

\section{METODOLOGIA}

Para emprender la investigación, se partió de las bases teórico-metodológicas propuestas en otras investigaciones, tal es el caso de la realizada por Trelles (2001). El diseño del procedimiento se enmarcó en:

- Los procedimientos existentes no están atemperados a las regulaciones vigentes para la actividad de comunicación organizacional en el país, entre las cuales se encuentran el Decreto Ley 252, la Resolución 60 y las Guías de Autocontrol actualizadas y 
- en la revisión bibliográfica no se encontraron procedimientos que integraran el sistema de comunicación con el resto de los sistemas de gestión, tal y como se establece en el requisito 7.4 de las últimas actualizaciones de las normas: NC ISO 9001, NC ISO 14001, NC ISO 45001 y NC ISO 56002 entre otras.

El procedimiento propuesto consta de seis fases y está dirigido al diagnóstico de la comunicación interna en cinco etapas (Fase I) y la externa en tres etapas (Fase II). Etapas de la Fase I:

- Etapa I. Enfoque de comunicación su correspondencia con: la historia, misión, imagen corporativa, valores y objetivos de la empresa.

- Etapa II. Cultura organizacional, clima interno, grado de implicación y sentido de pertenencia de los trabajadores.

- Etapa III. Características de los directivos y trabajadores: expectativas, grado de motivación, intereses, aspiraciones y participación en la dirección.

- Etapa IV. Naturaleza de los procesos de comunicación: redes, flujos, canales más usados, uso de los mensajes, frecuencia y su planificación.

- Etapa V. Tipo de comunicación predominante, relevancia y funciones de la comunicación.

La comunicación externa juega un papel de suma importancia en el procedimiento ya que, la determinación de los requisitos, necesidades y expectativas de los clientes y otras partes interesadas externas a la organización, así como la retroalimentación de la satisfacción de los servicios o productos, es el eje central del sistema de gestión de la calidad. A partir de la revisión bibliográfica se agrupa la misma en la Fase II en tres etapas:

- Etapa I. Comunicación externa operativa: Se produce diariamente con todas las partes interesadas externas de la organización: clientes, proveedores y otras partes interesadas, teniendo en cuenta la necesidad de conocer la imagen que puedan tener sobre la empresa, como base para el trabajo en la comunicación externa enfocada a promover la cartera de productos $y / 0$ servicios y la retroalimentación de la satisfacción de los mismos.

- Etapa II. Comunicación externa estratégica: El objetivo es obtener información sobre aspectos estratégicos de la organización que permitan mejorar su posición competitiva: estudio de la competencia, novedades legislativas, estado del mercado, vigilancia tecnológica, inteligencia empresarial, entre otros.

- Etapa III. Comunicación externa de notoriedad: Se refiere al flujo de información con el que la organización trata de dar a conocer sus productos y servicios y mejorar su imagen y reputación: prensa, publicitarios, revistas, registro de marcas, identidad visual corporativa, medios de comunicación tanto electrónicos, como impresos y audiovisuales a fin de poder garantizar un adecuado nivel de conocimiento y reconocimiento social sobre la empresa, sus resultados, sus trabajadores y sus valores.

En la Fase III del procedimiento se identifica el estado de la comunicación pertinente al resto de los sistemas de gestión según los requisitos establecidos en las normas técnicas y jurídicas de cada uno. En las normas ISO se refiere que la organización debe establecer, implementar y mantener los procesos necesarios para las comunicaciones internas y externas pertinentes a cada sistema y deben incluir: qué comunicar, cuándo comunicar, a quién comunicar, cómo comunicar y quién comunica.

En la Fase IV se diagnostica el tratamiento a las quejas y sugerencias de los trabajadores y de las partes interesadas externas de la organización, posteriormente en la Fase V, se identifica cómo se organiza: 
- La retroalimentación entre directivos y trabajadores en beneficio de la organización,

- la rendición de cuentas de las diferentes áreas de la empresa ante su consejo de dirección y trabajadores y

- la mejora continua de la comunicación organizacional.

Finalmente, en la Fase $\mathrm{VI}$, a partir de los problemas detectados en cada una de las fases anteriores se elabora un plan de acción con el objetivo de revertir aquellos aspectos de la comunicación identificados como nudos críticos.

En cada una de las fases y como parte de la realización del diagnóstico se procede al tratamiento de la temática abordada desde su comportamiento actual, las causas que la originan y sus consecuencias. Para este propósito se emplean entre otros los siguientes métodos y técnicas: análisis y síntesis, inducción- deducción, encuestas, entrevistas, listas de chequeo, observación directa, grupo focal y análisis documental. A continuación, se muestra una representación gráfica del procedimiento para su mejor comprensión.

Gráfico 1.

Procedimiento para el diagnóstico del Sistema de Comunicación

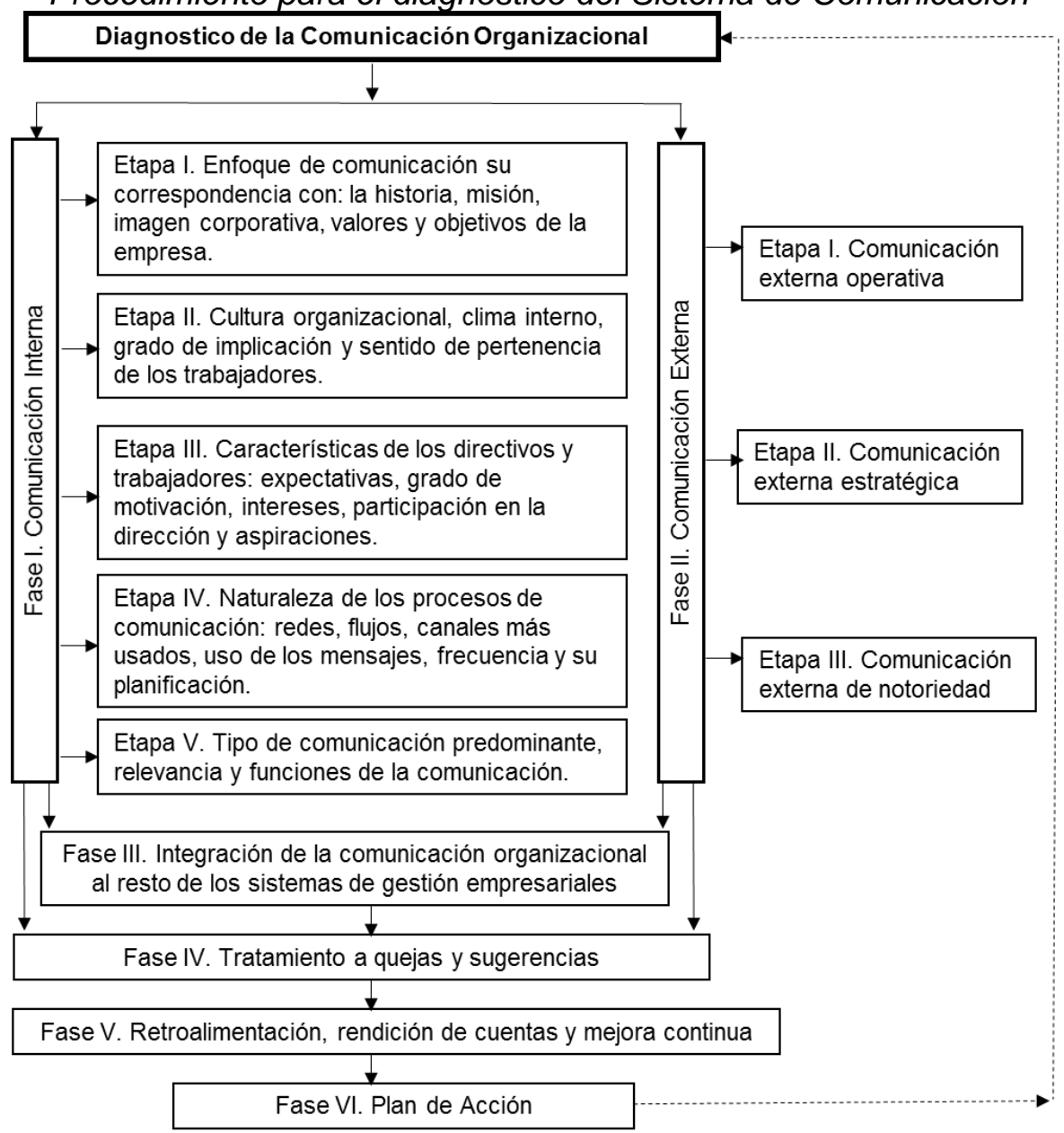

Para fundamentar, argumentar y evaluar el estado de la comunicación con el empleo del procedimiento representado en el Grafico 1, se diseñaron y aplicaron diferentes técnicas y herramientas de investigación entre las que se encuentran:

1. Listas de chequeo: Se elaboraron dos listas de chequeo. La primera permite la revisión de la implementación de la comunicación interna pertinente a la Gestión 
de los Recursos Humanos para verificar la conformidad o cumplimiento de las normas jurídicas vigentes que tributan a la actividad de comunicación entre la que se encuentra la Ley 116 y el Decreto 326.

Una segunda lista donde se verifica el cumplimiento de los requisitos que tributan a la comunicación interna y externa de los sistemas de gestión implementados de acuerdo a las normativas pertinentes a cada uno.

2. Guías de Autocontrol actualizadas del Sistema de Control Interno para el componente Información y Comunicación: En función del tipo de organización se selecciona la guía (empresarial o presupuestada), para lo cual se emplea: la revisión de documentos oficiales de la empresa, la observación directa y la realización de entrevistas a cuadros, especialistas y trabajadores.

3. Encuestas a los trabajadores: El cuestionario o encuesta elaborada se aplica a una muestra representativa de trabajadores, con representación de todas las áreas de la estructura organizativa y categorías ocupacionales, posteriormente se procesan estadísticamente. La encuesta es anónima y posee 55 preguntas enfocadas a conocer las opiniones acerca de aspectos relativos a la comunicación organizacional. El instrumento se divide según el tema o la necesidad, siempre subdividido por objetivos y temas precisos a diagnosticar.

Las preguntas son concisas y están elaboradas en un lenguaje claro y directo y abordan elementos propios de la organización que tributan a un sistema de comunicación eficaz.

4. Encuestas a directivos: Se aplican a los directivos y permiten identificar:

- Los tipos de canales que utilizan para comunicarse con sus colaboradores y como evalúan el empleo de los mismos y

- los valores que estos poseen, los que consideran que están presentes en la empresa y los que se deben fomentar.

5. Encuesta a clientes: Para el estudio de clientes se emplea la encuesta como herramienta de investigación, por considerar que la satisfacción del cliente es uno de los principales indicadores de la calidad de un servicio y con ello del adecuado empleo de los canales de comunicación para lograr dicha satisfacción, que surge de la diferencia entre las expectativas que se generan antes de contratar el servicio y lo que el cliente percibe una vez que la experiencia ha tenido lugar.

6. Revisión y análisis de documentos: Se revisan actas, documentos y otros registros que evidencian el cumplimiento de normas legales y técnicas vigentes aplicables a las actividades de la empresa, entre los que se encuentran:

- Actas de los Consejos de Dirección,

- actas de las reuniones de afiliados a las Secciones Sindicales,

- el Convenio Colectivo de Trabajo,

- el Reglamento Disciplinario Interno,

- el Plan de Capacitación y Desarrollo de la Empresa,

- la documentación del Sistema de Seguridad y Salud en el Trabajo,

- el expediente de Atención al Cliente (quejas y sugerencias),

- el procedimiento para la ejecución de los controles integrales en el año,

- los objetivos de Trabajo de la empresa,

- documentos pertinentes a cada sistema de gestión implementado.

Luego del análisis y procesamiento estadístico de las herramientas anteriormente enumeradas, de la interpretación de los datos, así como, el cruzamiento de las múltiples variables cualitativas que se miden, que parte de considerar los aspectos que para el diagnóstico de comunicación empresarial integrado se plantean en las 
normativas vigentes, se abordan los resultados agrupándolos en las seis Fases del procedimiento.

\section{RESULTADOS}

Para validar el procedimiento propuesto por los autores, el mismo fue aplicado en dos organizaciones de Santiago de Cuba, ellas fueron:

- Centro de Información y Gestión Tecnológica de Santiago de Cuba, conocido en el territorio por su marca comercial, MEGACEN adscrito al Instituto de Información Científica y Tecnológica, perteneciente a la Agencia de Energía Nuclear y Tecnologías de Avanzadas del Ministerio de Ciencia, Tecnología y Medio Ambiente (CITMA) es una Entidad de Ciencia, Tecnología e Innovación (ECTI) que se encarga como centro de interfase de la prestación de servicios científicos-técnicos mediante acciones de transferencia tecnológica y transferencia de conocimiento a las organizaciones del territorio que les permitan cumplir con las políticas del país y la actualización del modelo económico cubano para lograr la sostenibilidad económica. Su Misión es: Elevar la competitividad de nuestros clientes, brindando servicios integrales y especializados de información y consultoría, utilizando las nuevas tecnologías de la información y el conocimiento.

Entre los objetivos de trabajo trazados por MEGACEN para lograr el desarrollo sostenible del territorio está el trabajo con las diferentes formas de gestión que existen hoy en la economía cubana mediante el uso intensivo del conocimiento y la innovación apoyándolas en sus procesos de producción de bienes o prestación de servicios.

- Empresa Ferrocarriles de Oriente (EFO) perteneciente a la OSDE Unión de

Ferrocarriles de Cuba del Ministerio del Transporte, ubicada en la Avenida Jesús Menéndez $s / n$ en el municipio Santiago de Cuba. El diagnóstico realizado, se enmarcó en la oficina central de la empresa que está compuesta por 10 direcciones funcionales y 8 departamentos adscritos al director general que es el máximo responsable del Sistema de Comunicación Empresarial.

En el artículo de Domínguez Quevedo, L. \& Suarez Caimary, I. L. (2021) se muestran los resultados obtenidos con la aplicación del procedimiento propuesto en este estudio en la EFO.

La EFO tiene la Misión de: Garantizar, con eficiencia y calidad, las transportaciones de cargas especializadas, carga general y pasajeros para la satisfacción de las necesidades de otras entidades y de la población y de esa forma contribuir al desarrollo socio- económico del país.

Y como Visión: Constituir, a mediano plazo, una entidad bien organizada, eficaz, altamente profesional y competitiva que promueva la cooperación para garantizar progresivamente el incremento de la eficiencia y la excelencia en el cumplimiento de los requerimientos de calidad.

En los Consejos de Dirección de las dos entidades se presentaron los resultados arrojados luego de realizado el diagnóstico de la comunicación integrado, constituyendo esto un importante paso para la mejora de la gestión organizacional en estas prestigiosas entidades de la provincia ya permite el diseño e implementación del sistema de comunicación con enfoque estratégico e integrado. Se logra la satisfacción total por parte de los clientes con el estudio realizado.

En el Plan de Acciones propuesto en ambas entidades (Fase VI del procedimiento), se abarcaron las dificultades y problemáticas diagnosticadas en las 
diferentes fases del procedimiento respondiendo a los indicadores relacionados con la comunicación en las normativas aplicables vigentes.

\section{DISCUSION}

Se logra imbricar los elementos teóricos, metodológicos, estructurales y prácticos a tener en cuenta en un diagnóstico de comunicación para el diseño e implementación del sistema de comunicación, que permite su integración con los sistemas de gestión organizacionales para lograr la mejora de los procesos empresariales y la satisfacción de las partes interesadas.

El procedimiento fue aplicado y validado con satisfacción total en dos entidades del territorio empleando el procesamiento y análisis de información, en función de las técnicas y herramientas de investigación utilizadas.

Se recomienda su perfeccionamiento continuo, a partir de los resultados obtenidos con su aplicación, como parte de la mejora continua.

Se concluye que el modelo propuesto atemperado a las normas jurídicas y técnicas vigentes con enfoque estratégico, integrado, participativo y sistémico para la actividad de comunicación permite su empleo o generalización a cualquier tipo de organización, ya sea de producción de bienes materiales o de prestación de servicios.

\section{REFERENCIAS BIBLIOGRÁFICAS}

Almirall, A. (2010). Metodología para el diagnóstico del proceso de comunicación organizacional de la Oficina Central de QUIMEFA", trabajo de diploma. La Habana. Instituto Superior Politécnico José Antonio Echeverría. Centro de Estudios de Técnicas de Dirección. Facultad de Ingeniería Industrial.

Bartoli, A. (1992). Comunicación y Organización. Barcelona. Editorial Paidós.

Cirigliano, C. (2017). Gestión de la Comunicación Interna en las Organizaciones: hacia un marco teórico y definición de herramientas de trabajo. Argentina. http://www.monografias.com/trabajos15/comunic-interna/comunicinterna.shtmlte

Cuba. Asamblea Nacional del Poder Popular (2014). Ley 116 Código de Trabajo. Gaceta Oficial de la República de Cuba, No. 29 Extraordinaria. http://www.gacetaoficial.gob.cu

Cuba. Consejo de ministro (2014). Decreto 326 Reglamento del Código de Trabajo. Gaceta Oficial de la República de Cuba, No. 29 Extraordinaria. http://www.gacetaoficial.gob.cu

Cuba. Consejo de Estado (2018). Decreto Ley No. 252 Sobre la Continuidad y el Fortalecimiento del Sistema de Dirección y Gestión Empresarial Cubano. Gaceta Oficial de la República de Cuba, No. 56 Ordinaria. http://www.gacetaoficial.gob.cu

Cuba. Consejo de Ministro (2018). Decreto No. 281 Reglamento para la Implantación y Consolidación del Sistema de Dirección y Gestión Empresarial Estatal. Gaceta Oficial de la República de Cuba, No. 56 Ordinaria. http://www.gacetaoficial.gob.cu 
Cuba. Contraloría General de la República (2011). Resolución 60 Normas del Sistema de Control Interno. http://www.fgr.gob.cu/sites/default/files/Resolucion\%2060.pdf

Cuba. Oficina Nacional de Normalización (2015). Norma cubana ISO 14001: 2015, Sistema de Gestión Ambiental - Requisitos con orientación para su uso. La Habana, Cuba: autor.

Cuba. Oficina Nacional de Normalización (2015). Norma cubana ISO 9001: 2015, Sistema de Gestión de la Calidad - Requisitos con orientación para su uso. La Habana, Cuba: autor.

Cuba. Oficina Nacional de Normalización (2018). Norma cubana ISO 45001: 2018, Sistema de Gestión de la Seguridad y Salud en el trabajo - Requisitos con orientación para su uso. La Habana, Cuba: autor.

Cuba. Oficina Nacional de Normalización (2020). Norma cubana ISO 56002: 2020, Gestión de la Innovación - Orientación. La Habana, Cuba: autor.

Cuba. Contraloría General República de Cuba (2020). Guía de Autocontrol General Actualizada. http://www.fgr.gob.cu

Domínguez Quevedo, L. \& Suarez Caimary, I. L. (2021). Metodología para el diagnóstico integral de la comunicación empresarial. Caso de estudio Empresa Ferrocarriles de Oriente. LUZ, 20(3), 22-33. https://luz.uho.edu.cu/index.php/luz/article/view/1123

Dong, Z. \& Morales Rodríguez, E. (2013). Diseño e implantación del sistema de comunicación en una Empresa de Tabaco Torcido. https://www.monografias.com/trabajos99/diseno-e-implantacion-del-sistemacomunicacion-empresa-tabaco-torcido/diseno-e-implantacion-del-sistemacomunicacion-empresa-tabaco-torcido2.shtml

Llanas, J. (2021). Sistemas de Comunicación. Economipedia. https://economipedia.com/definiciones/sistemas-de-comunicacion.html

Núñez, Y., Rodríguez, C., Arancibia, S. (2010). Modelo de gestión por procesos para la comunicación organizacional como recurso intangible en instituciones de Educación Superior. En Eighth LACCEI Latin American and Caribbean Conference for Engineering and Technology (LACCEl'2010) (June 1-4, 2010). Arequipa, Perú. Innovation and Development for the Americas. http://www.laccei.org/LACCEI2010-

Peru/Papers/Papers_pdf/UM053_Nunez.pdf

Trelles, I. (2001). Comunicación Organizacional. La Habana. Editorial Félix Varela. 